\title{
Safety of a GFD in Pregnant Women Without Celiac Disease: Investigating Ingrained Habits
}

\author{
Javier A. Villafuerte Gálvez ${ }^{1,2}$. Jocelyn A. Silvester ${ }^{1,2,3}$ \\ Published online: 10 July 2020 \\ (c) Springer Science+Business Media, LLC, part of Springer Nature 2020
}

People without celiac disease avoiding gluten (PWAGs) have increased as a proportion of the US population from an estimated $0.5 \%$ in 2009 to $1.7 \%$ in 2014 . PWAGs thus exceed by more than twofold the estimated $0.7 \%$ of people diagnosed with celiac disease $(\mathrm{CeD})$ [1]. Although PWAGs may avoid gluten for multiple reasons, including perceived health benefits [2], and adopting the diet to support a relative with $\mathrm{CeD}$ or non-celiac wheat/gluten sensitivity, their motivations are neither well characterized nor well understood. For those with celiac disease, gluten avoidance improves symptoms and nutritional deficiencies and decreases chronic small intestinal inflammation, thereby reducing the likelihood of long-term complications such as osteoporosis or gastrointestinal malignancies. Nonetheless, this substantial benefit comes at the cost of following a difficult [3] and often costly [4] diet. Even in patients with CeD, a gluten-free diet (GFD) can have adverse effects, such as micronutrient deficiencies, constipation, and weight gain [5].

In people without $\mathrm{CeD}$, claims of the health benefits of gluten avoidance have spread more rapidly than the evidence of its potential adverse effects. These unsubstantiated health claims along with a booming gluten-free (GF) food market are likely driving gluten avoidance among the general population. It is in this context that in this issue of Digestive Diseases and Sciences Wagner et al. [6] ask whether pregnant PWAGs have maternal and fetal outcomes different from those of their gluten-eating peers.

The design chosen to answer this question is a retrospective study of two cohorts from a single institution. Inclusion

Javier A. Villafuerte Gálvez

jvillaf1@bidmc.harvard.edu

1 Harvard Celiac Research Program, Harvard Medical School, Boston, MA, USA

2 Digestive Disease Center, Beth Israel Deaconess Medical Center, Boston, MA, USA

3 Division of Gastroenterology, Hepatology and Nutrition, Boston Children's Hospital, Boston, MA, USA into the childbearing PWAG cohort $(n=138)$ required documentation of gluten avoidance during a prenatal visit or the request for a GFD during admission for labor and delivery (L\&D). The control cohort consisted of age-, sex-, and ethnicity-matched childbearing women at the same institution who did not meet the inclusion criteria for the PWAG cohort. Exclusion criteria for both groups included chart documentation, serology, or duodenal histology consistent with $\mathrm{CeD}$, wheat allergy, or dermatitis herpetiformis. Maternal and fetal outcomes of interest were recorded and compared between the two groups.

Both study cohorts were carefully defined in an attempt to exclude pregnant women with a known history, serology, or histology of $\mathrm{CeD}$ based on institutional records. This approach, as rightly acknowledged in the discussion section, could miss patients diagnosed with $\mathrm{CeD}$ outside of the hospital network or who have the disease without a formal diagnosis but who have decided to avoid gluten. That concern is intensified by relatively high frequencies of hypothyroidism (24.6\% vs. $10.1 \%)$ and chart diagnosis of IBS (8.7\% vs. $1.45 \%$ ) in PWAGs compared to controls. Patients with $\mathrm{CeD}$ have an increased risk of hypothyroidism [7] and can easily be misdiagnosed with IBS [8]. Other explanations for this imbalance remain plausible. Information on the outcomes of patients with $\mathrm{CeD}$ excluded from these cohorts could have been of great interest as a group that is best known to benefit from GFD during pregnancy [9]. It is a missed opportunity to attempt to differentiate the effects of a GFD from those of $\mathrm{CeD}$.

Important maternal outcomes such as gestational weight gain, type of delivery, indication for delivery, gestational hypertension, preeclampsia, gestational diabetes, postpartum depression, and postpartum transfusion requirement did not differ significantly between PWAG and control mothers. Similarly, the composite rate of all neonatal complications did not differ between the PWAG and the control group ( $44.2 \%$ vs. $42.8 \%, p=0.82$ ). Key neonatal outcomes including gestational age at delivery, birth weight, Apgar 
scores, major fetal malformations, demise, hyperglycemia, jaundice, respiratory distress syndrome, and need for intensive care were analyzed independently and did not differ between groups.

The study by Wagner et al. provides novel and useful information for gastroenterologists and other clinicians who may be asked by patients without $\mathrm{CeD}$ for advice regarding gluten avoidance during pregnancy. Yet, the apparent good news must be balanced by its description of a relatively narrow and specific population composed mostly of commercially insured White non-Hispanic women in the New York City area. One could theorize this population may be better equipped to mitigate potentially adverse effects of a GFD. For example: (1) they may have better access to a wider variety of nutritious GF foods that they can afford; (2) they may have superior access to medical care, expert nutritional advice, and thus better adhere to antenatal micronutrient supplementation; and (3) they may have available more time, space and opportunity for physical activity in order to avoid weight gain.

It is also essential to consider the results in the context of limited information resulting in substantial uncertainty regarding the "dose" of the GFD-intensity (adherence), duration, and timing related to pregnancy. Over a quarter $(69 / 245,28.2 \%)$ of patients initially screened to be in the PWAG group were included based on having an inpatient order for a GFD on admission to L\&D. It is possible that they avoided gluten even before their pregnancy or only in the immediate peripartum setting. This "dose gradient" in the independent variable has considerable implications for the strength of the conclusions as effects of a nutritionally imbalanced GFD may take time to accrue. Moreover, the physiology of embryonic and fetal development would suggest the timing and duration of gluten avoidance to be of vital relevance if it led to deficiencies of essential micronutrients such as iron and folate. Folate deficiency during the first 4 weeks of pregnancy predisposes to neural tube defects [10]. Whereas iron deficiency is most important in the third trimester as the rapid expansion of the placenta, fetus and maternal blood pool considerably increase the iron requirements [11]. Perhaps, subgroup analysis for important outcomes of the study between those recruited by antenatal intake forms as opposed to inpatient dietary orders could dispel these concerns and even help validate an inpatient dietary order as an appropriate proxy for recruitment for future similar studies.

The maternal and fetal outcomes chosen by the authors seem relevant and appropriate. Fetal adverse outcomes such as preterm birth or small for gestational age are uncommon in the developed world, with frequencies around 5-7\% [9]. While the sample size could permit detection of changes of two to fourfold in these frequencies with a power of $80 \%$, the authors sensibly chose to generate a composite outcome of neonatal complications enabling the detection of changes of $\sim 33 \%$ in the rate of overall neonatal complications (post hoc calculations based on OpenEpi Software [12]).

There is generally a lack of data regarding the effects of diets commonly prescribed for digestive ailments (e.g., low FODMAP, carbohydrate exclusion diets for inflammatory bowel disease) in special populations, such as pregnant women, children, and the elderly. Increasing interest from the general population and the willingness of gastroenterologists to consider dietary manipulation in the management of common GI conditions necessitates further research about the effects of such diets not only in special populations, but also with long-term use/adherence.

It is in this context that we welcome the arrival of Wagner et al.'s study and congratulate them on their delivery of useful evidence regarding dietary interventions that are inherently difficult to control in a randomized fashion. Despite concerns about generalizability, the study population may be representative of many reproductive-age PWAG women considering pregnancy in the USA [1]. For now, recommendations regarding a GFD during pregnancy in a non-celiac patient should remain individualized, emphasize adherence to standard recommendations for prenatal micronutrient supplementation, and start with a shared understanding of how little we know about potential risks and benefits.

Funding JAVG is funded by the Research Training Grant in Gastroenterology 2T32DK007760-16. JAS is supported by the National Institute of Diabetes and Digestive and Kidney Diseases of the National Institutes of Health under Award Number K23DK119584. The content is solely the responsibility of the authors and does not necessarily represent the official views of the National Institutes of Health.

\section{Compliance with Ethical Standards}

Conflict of interest JAVG declares no financial conflict of interest. JAS has received research support from Biomedal S.L., Cour Pharma, Glutenostics L.L.C., Milky Way Life Sciences, and the Celiac Disease Foundation and has served on an advisory board for Takeda Pharmaceuticals.

\section{References}

1. Choung RS, Unalp-Arida A, Ruhl CE, et al. Less hidden celiac disease but increased gluten avoidance without a diagnosis in the USA: findings from the National Health and Nutrition Examination Surveys from 2009 to 2014. Mayo Clin Proc. 2016. https:// doi.org/10.1016/j.mayocp.2016.10.012.

2. Niland B, Cash BD. Health benefits and adverse effects of a gluten-free diet in non-celiac disease patients. Gastroenterol Hepatol. 2018;14:82.

3. Shah S, Akbari M, Vanga R, et al. Patient perception of treatment burden is high in celiac disease compared to other common 
conditions. Am J Gastroenterol. 2014;109:1304-1311. https://doi. org/10.1038/ajg.2014.29.

4. Villafuerte-Galvez J, Vanga RR, Dennis M, et al. Factors governing long-term adherence to a gluten-free diet in adult patients with coeliac disease. Aliment Pharmacol Ther. 2015;42:753-760. https ://doi.org/10.1111/apt.13319.

5. Kabbani TA, Goldberg A, Kelly CP, et al. Body mass index and the risk of obesity in coeliac disease treated with the gluten-free diet. Aliment Pharmacol Ther. 2012;35:723-729. https://doi.org /10.1111/j.1365-2036.2012.05001.x.

6. Wagner BA, Zork N, Blackett JW, et al. Characteristics and maternal-fetal outcomes of pregnant women without celiac disease who avoid gluten. Dig Dis Sci. (Epub ahead of print). https://doi. org/10.1007/s10620-020-06232-3.

7. Counsell CE, Taha A, Ruddell WS. Coeliac disease and autoimmune thyroid disease. Gut. 1994;35:844-846.

8. Card TR, Siffledeen J, West J, et al. An excess of prior irritable bowel syndrome diagnoses or treatments in Celiac disease: evidence of diagnostic delay. Scand J Gastroenterol. 2013;48:801807. https://doi.org/10.3109/00365521.2013.786130.
9. Saccone G, Berghella V, Sarno L, et al. Celiac disease and obstetric complications: a systematic review and metaanalysis. Am J Obstet Gynecol. 2016;214:225-234. https://doi.org/10.1016/j. ajog.2015.09.080.

10. Cavalli P. Prevention of neural tube defects and proper folate periconceptional supplementation. J Prenat Med. 2008;2:40-41.

11. Scholl TO. Maternal iron status: relation to fetal growth, length of gestation and the neonate's iron endowment. Nutr Rev. 2011;69:S23-S29. https://doi.org/10.111 1/j.1753-4887.2011.00429.x.

12. Dean AG, Sullivan KM, Soe MM. OpenEpi: open source epidemiologic statistics for public health. 2013. https://www.opene pi.com/Menu/OE_Menu.htm. Accessed March 27, 2020.

Publisher's Note Springer Nature remains neutral with regard to jurisdictional claims in published maps and institutional affiliations. 